\title{
Event-Specific Risk Factors Predicting Episodes of Unprotected Anal Intercourse with Male Nonregular Partners among Men Who Have Sex with Men Using Case-Crossover Study Design
}

\author{
Jinghua Li, ${ }^{1}$ Joseph T. F. Lau, ${ }^{1,2,3,4}$ Jing Gu, ${ }^{5}$ Chun Hao, ${ }^{5}$ and Coco H. Y. Lai ${ }^{1}$ \\ ${ }^{1}$ School of Public Health and Primary Care, Faculty of Medicine, The Chinese University of Hong Kong, \\ Shatin, Hong Kong \\ ${ }^{2}$ CUHK Shenzhen Research Institute, Shenzhen 518057, China \\ ${ }^{3}$ Centre for Medical Anthropology and Behavioral Health, Sun Yat-sen University, Guangzhou 510080, China \\ ${ }^{4}$ Centre for Health Behaviors Research, School of Public Health and Primary Care, Faculty of Medicine, \\ The Chinese University of Hong Kong, 5/F., School of Public Health, Prince of Wales Hospital, Shatin, New Territories, Hong Kong \\ ${ }^{5}$ School of Public Health, Sun Yat-sen University, Guangzhou 510080, China
}

Correspondence should be addressed to Joseph T. F. Lau; jlau@cuhk.edu.hk

Received 7 May 2014; Revised 6 July 2014; Accepted 6 July 2014; Published 20 July 2014

Academic Editor: Esteban Martinez

Copyright (C) 2014 Jinghua Li et al. This is an open access article distributed under the Creative Commons Attribution License, which permits unrestricted use, distribution, and reproduction in any medium, provided the original work is properly cited.

\begin{abstract}
This study investigated event-specific factors that determine episodes of unprotected and protected anal intercourse (UAI and PAI) among 215 men who have sex with men (MSM), who used condoms inconsistently with nonregular partners (NRP) in the last six months, in Hong Kong. A case-crossover study design was used. Lower likelihood of episodes involving UAI with NRP was associated with (1) five partner attributes (NRP were $<35$ years old, at least three previous anal sex experiences with the NRP, perception that participant and the NRP had asymmetrical sexual experience, perception that the NRP was feminine, and liking toward the NRP; OR=0.16-0.52), (2) six situational variables (the participant having had UAI with another man in the last week, having discussed condom use, perception that the NRP liked to use condom, partner's suggestion to have PAI, participant's suggestion to have PAI, and participant's plan to use condoms; OR =0.11-0.39), and (3) four environmental/setting variables (condoms already placed at the venue, display of condom use promotion materials, participant's possession of a condom, and the NRP possessed a condom; OR $=0.27-0.45$ ). HIV prevention targeting MSM should focus on event-specific protective factors, which may be different from those obtained from studies distinguishing condom users versus nonusers.
\end{abstract}

\section{Introduction}

In many Asian cities, HIV prevalence among men who have sex with men (MSM) has been increasing sharply [1]. In China, a 61-city study documented HIV prevalence of $4.9 \%$ in 2009 [2]. The incidence of HIV was high in China, 5.1 and 5.4 per 100 person-years in Nanjing [3] and Shenyang [4], respectively. In 2008, the HIV prevalence among MSM in Hong Kong was 4.3\% [5]. Specifically, $84.6 \%$ of the MSM in Hong Kong had had sex with nonregular partners (NRP) in the last six months; of those MSM, $27.9 \%$ had had unprotected anal intercourse (UAI) with a NRP [6].
Globally, high percentages of MSM have NRP, and UAI is often involved [7-9]. Factors associated with UAI are often reported and serve as basis for forming HIV interventions to reduce UAI $[10,11]$. The factors that specifically apply to NRP among MSM include low education level [12], low level of HIV-related knowledge [12], recruitment of male sex partners at gay venues [9], lack of exposure to HIV prevention services [7], and concern about acquiring sexually transmitted diseases [8]. Factors associated with UAI with NRP among MSM in Hong Kong include perceived chance of contracting HIV, having had anal sex with more than six partners, perceived nonavailability of condoms, and sourcing 
NRP from brothels [6]. In these studies, the participants who were MSM were divided into a group of men ever had had UAI and never had had UAI in the past month or past few months. Cross-sectional analysis is commonly used to distinguish between these two groups.

Another research question is, however, important to HIV prevention. Instead of what mentioned above, the question focuses only on the group of inconsistent condom users, that is, those who sometimes had UAI and sometimes had protected anal intercourse (PAI). The research question is "Why do these inconsistent condom users sometimes use condoms and sometimes not?" or "Are the situations different when UAI occurs and does not occur?" This new research question cannot be answered by traditional cross-sectional analysis and logistic regression analysis, as it involves only one group of MSM (those who were inconsistent condom users). It can, however, be answered by a relatively new study design, the case-crossover design, which can be used to identify situational predictors of UAI within the same individuals, who sometimes but not always use condoms during anal intercourse (inconsistent condom users). In our context, the design compared presence of situational factors in two episodes of anal intercourse, the last episode of UAI and the last episode of PAI. The matched (conditional) logistic regression method is a standard method to analyze such data. In our case, a pair of episodes of UAI and PAI of the same individual is used as unit of analysis $[13,14]$. Hence, it allows researchers to investigate various event-specific risk factors to distinguish episodes of UAI from episodes of PAI.

The literature shows that the case-crossover study design is able to delineate the temporal relationship between variables of interest and has better control for confounding factors (e.g., participants' characteristics), since the information is obtained from the same individuals [15]. Thus, it is a compelling approach for investigating a causal pathway [16] and can minimize biases due to measured and unmeasured time-invariant potential confounders since every sampled individual serves as his own control [17]. The case-crossover analysis has important implications in HIV prevention, as it draws health workers' attention to situational factors that lead to UAI among inconsistent condom users, a group that may benefit more than consistent condom users from HIV prevention programs. Although the case-crossover design is a relatively new method, it is well established and has been used in studying different types of health-related behaviors (e.g., alcohol and drug use, first-time sexual encounter, and sex at home). A dearth of studies has, however, used this type of design to answer the aforementioned research question about HIV prevention.

The present study applied the case-crossover study design to investigate whether three broad types of event-specific factors are predictive of episodes of UAI among MSM who were inconsistent condom users during anal sex with male NRP in Hong Kong: (1) factors related to the sex partner's attributes (e.g., age and physical appearance), (2) situational factors (e.g., appraisal of the risk of the NRP, communication with the NRP about condom use, sexual compulsivity, and use of alcohol), and (3) environmental/settings factors (e.g., timing and location of the sex episode, availability, and possession of condoms).

\section{Methods}

2.1. Study Design. Inclusion criteria were (1) Hong Kong Chinese men from 18 to 60 years old (those holding a Hong Kong identification card), who have (2) had both at least one episode of UAI with a NRP and at least one episode of PAI with a NRP (i.e., an inconsistent condom user) in the last six months. A mapping exercise was conducted by the government and identified 12 gay bars and 16 gay saunas in Hong Kong. Approval was obtained from six gay bars and ten gay saunas.

The venue-based interviews were administered by a group of experienced and trained peer interviewers. Participants were also recruited from two public beaches that were favored by gay men. This combined method has been used to recruit MSM study participants [18-20]. Peer interviewers visited these venues at different time slots during weekdays and weekends. They briefed prospective participants about the study and invited them to join the study. Venue-based sampling was performed from September 2010 to June 2011. Several screening questions were asked to establish the participants' eligibility and verbal informed consent was obtained before the face-to-face interview commenced in settings with privacy ensured. Interviewers signed a form pledging that they had explained information contained in the information sheet clearly to the participant. Upon completion of the interview, participants were given a coupon of $\mathrm{HK} \$ 50$ (about 6 USD) cash value, to compensate their time spent for the interview.

In addition to venue-based sampling, some participants were recruited through the internet, since some MSM did not attend gay venues but are accessible through the internet. Previous studies have shown that many MSM recruit friends and sexual partners from the internet make recruiting this population an important step for understanding HIV-related risk behaviors. The mixed method has been used in many published studies on sexual health of MSM, including a number of local studies [18, 20] and others conducted outside Hong Kong [21, 22], and enabled us to recruit MSM of different characteristics. Banners were posted on four gay websites, which were frequently visited by MSM in Hong Kong. The websites have functions of chat rooms, partner finding, and provision of information (addresses of local gay venues) and are well known to MSM in Hong Kong. Since the recruitment method was different from that of the venue-based sampling, verbal informed consent was not feasible for the internet-based recruitment which was not administered by fieldworkers. Instead, participants were provided with information at the beginning of the online questionnaire, which briefed them about the voluntary nature, background, and purposes of the study and told that the return of the completed internet-based questionnaire to the researchers implied provision of their informed consent [18-20]. The internet-based participants then selfadministered the anonymous internet-based questionnaire, 
which was identical to the one used in the venue-based survey. Participants were able to claim a supermarket coupon of $\mathrm{HK} \$ 50$ cash value by providing a mailing address (without including their names). The envelops did not include any name nor information about HIV; the mailing of coupons as an incentive for completing surveys has been performed in other published studies on MSM $[23,24]$. We cross-checked that there were no overlapping addresses provided to us. A total sample size of 215 was achieved (151 venue-based interviews and 64 internet-based questionnaires).

\subsection{Measures}

2.2.1. Background Characteristics of the Participants. Data on sociodemographic variables, sexual orientation, and the mode of recruitment were collected.

2.2.2. Last Episodes of UAI and PAI with Male NRP. Participants reported the presence or absence of an event-specific factor during the most recent episode of UAI with a NRP and the most recent episode of PAI with a NRP. (e.g., "Did you use alcohol before the last episode of UAI with a male NRP?" or "Did you use alcohol before the last episode of PAI with a male NRP?"). As mentioned, these data were analyzed in paired data of the same individuals. Operationally, a NRP was defined as "one whom you met casually for the purpose of having sex but did not involve money."

2.2.3. Event-Specific Factors Related to Attributes of the Nonregular Male Sex Partners (NRP). Information collected on NRP included (1) sociodemographic characteristics, (2) sexual experiences, (3) style of the partner (i.e., physical appearance being masculine, feminine or neutral, and perceived personality of the NRP being assertive, passive, or inbetween), and (4) degree of liking (i.e., to which extend is the NRP attractive to the participant, how much the participant liked the NRP, and how much the participant perceived that the NRP liked him, on a scale from 1 "did not like" to 10 "liked very much") and relationship with the NRP (i.e., how the participant rates the relationship with the NRP using a 5 -point Likert scale from "very good" to "very bad").

2.2.4. Situational Variables. Information gathered about the situation surrounding UAI and PAI included (1) the participant's spirit prior to the episode of anal sex (mood (how did the participant feel prior to having anal sex?), tiredness (whether the participants felt tired), and whether the participants felt nervous), (2) risk assessments (e.g., perceived risks of HIV and STI of the NRP), (3) communication and planning about condom use (e.g., discussion with the NRP about condom use and condom use being suggested by the participant), (4) sexual behavioral variables (e.g., sexual compulsivity and sexual position in the last episode of UAI and PAI and sexual dysfunctions of no sexual desire, erectile dysfunction, premature ejaculation, and pain during intercourse during the previous month), and (5) substance use before anal intercourse took place (alcohol, potency drugs, and psychoactive substances).
2.2.5. Event-Specific Environmental and Settings Variables. These variables included (1) timing and location of the sexual episodes (e.g., Hong Kong or overseas, weekend or weekday, at home or not at home), (2) perceived availability of condoms and display of reminders promoting condom use, and (3) physical conditions (i.e., whether the anal sex took place in a dark environment, whether there was shower facilities, and whether the hygiene of the place was relatively poor).

2.3. Statistical Analysis. As mentioned, data of the casecrossover design were organized and analyzed in pairs (i.e., presence or absence of the situational factors in the last UAI and last PAI episodes) which were matched within the same individuals. By matching, it means pairing of data. The presence or absence of a situational factor referring to the UAI episode was matched to (or paired with) those of the PAI episode. The data set was rearranged so that there were 215 pairs of data. For instance, for the situational factor of alcohol use, the paired data for the UAI and PAI episodes could be (yes, yes), (yes, no), (no, yes), and (no, no). It therefore refers to internal matching of all situational factors within the same individual. Data of all situational factors were organized and analyzed. To account for such pairing, conditional logistic regression, a standard method for the case-crossover design $[13,14]$, was used to assess the significance of the associations between the studied event-specific risk factors and occurrence of UAI. Matched odds ratios (OR) and their 95\% confidence intervals (CI) were presented. Since potential confounders of individuals' characteristics have been removed by pairing data of situation factors within the same individuals, such analyses do not need to adjust for participants' background characteristics [13]. Univariate matched OR are hence reported for each variable. The software PROC PHREG (SAS Institute, Cary, NC, release 9.2) was used. All other statistical analyses were performed using SPSS 18.0 (SPSS Inc., Chicago, IL). All statistical tests involved were two-tailed and a $P$ value $<0.05$ was considered statistically significant.

\section{Results}

3.1. Descriptive Statistics. About $3 / 4$ (74\%) of the participants were 35 years old or younger; $62.8 \%$ had attended a college or university; $87.4 \%$ identified as homosexual (see Table 1). No significant differences in sociodemographic variables were found between participants recruited from gay venues and via the internet $(P>0.1$, see Table 1$)$.

The majority of NRP were of 35 years old or younger (about 70\%), Hong Kong residents with Hong Kong identification cards $(>80 \%)$, and perceived by most participants (about $87 \%$ ) as attractive. About $50 \%$ of the NRP were described by the participant as having appearance which looked masculine and perceived to have an assertive personality. Over half $(57.7 \%)$ of the NRP met the participant on the same day and $68.6 \%$ had never had anal sex with the participant in the past (see Table 2). 
TABLE 1: Background characteristics of participants.

\begin{tabular}{|c|c|c|c|c|}
\hline & $\begin{array}{c}\text { All } \\
\%(n=215)\end{array}$ & $\begin{array}{l}\text { Recruited from gay venues } \\
\qquad \%(n=151)\end{array}$ & $\begin{array}{c}\text { Recruited from internet } \\
\%(n=64)\end{array}$ & $P$ value \\
\hline \multicolumn{5}{|l|}{ Sociodemographic characteristics } \\
\hline \multicolumn{5}{|l|}{ Age (years) } \\
\hline$\leq 30$ & 53.0 & 49.7 & 60.9 & \\
\hline $31-40$ & 34.4 & 34.4 & 34.4 & \\
\hline $41-50$ & 9.3 & 11.3 & 4.7 & \\
\hline$\geq 51$ & 3.2 & 4.6 & 0.0 & 0.105 \\
\hline \multicolumn{5}{|l|}{ Education level } \\
\hline Junior high or lower & 7.4 & 7.9 & 6.3 & \\
\hline Senior high & 29.8 & 31.1 & 26.6 & \\
\hline College and above & 62.8 & 60.9 & 67.2 & 0.681 \\
\hline \multicolumn{5}{|l|}{ Current marital status } \\
\hline Single & 87.4 & 86.0 & 92.2 & \\
\hline Cohabiting with a man & 7.9 & 8.0 & 7.8 & \\
\hline Cohabiting with or married to a woman & 3.8 & 5.3 & 0.0 & \\
\hline Divorced/widowed/others & 1.0 & 0.7 & 0.0 & 0.257 \\
\hline \multicolumn{5}{|l|}{ Sexual orientation } \\
\hline Homosexual & 87.4 & 87.4 & 87.5 & \\
\hline Bisexual & 10.7 & 9.9 & 12.5 & \\
\hline Heterosexual/uncertain & 1.9 & 2.6 & 0.0 & 0.373 \\
\hline \multicolumn{5}{|l|}{ Mode of recruitment } \\
\hline Bar or disco & 30.2 & & & \\
\hline Sauna & 35.8 & & & \\
\hline Internet & 29.8 & & & \\
\hline Beach & 4.2 & & & \\
\hline
\end{tabular}

${ }^{\#}$ By chi-square test.

Since there are many situational and environmental factors included in this study, we only describe the frequency distributions of those variables that were found to be significant in the conditional logistic regression that are described in Table 5, while the frequency distributions of all factors are listed in Tables 3 and 4 . The prevalence of presence of the significant situational and environmental factors in the UAI episode and PAI episode was being in an energetic or very energetic state prior to the episode of anal sex (UAI episode: $42.4 \%$; PAI episode: $46.0 \%$ ), nervous feeling prior to the episode of anal sex (UAI episode: $57.2 \%$; PAI episode: 50.3\%), having had UAI with another man one week prior to having sex with the NRP of the last episode anal sex (UAI episode: $14.4 \%$; PAI episode: $20.9 \%$ ), perception that the NRP would be unlikely to (or never) use condoms if they were going to have anal sex with other men (UAI episode: 49.8\%; PAI episode: $41.4 \%$ ), discussion about condom use prior to having had anal sex with the NRP (UAI episode: $21.4 \%$; PAI episode: $37.2 \%$ ), perception that the NRP would like to use condom (UAI episode: $15.8 \%$; PAI episode: $50.7 \%$ ), suggestion made by the NRP to use condom (UAI episode: $16.3 \%$; PAI episode: $34.0 \%$ ), suggestion made by the NRP to have UAI (UAI episode: $24.2 \%$; PAI episode: $13.0 \%$ ), plan made by the participant to use condom (UAI episode: $54.9 \%$; PAI episode: $81.9 \%$ ), participant's suggestion to use condom (UAI episode: $20.0 \%$; PAI episode: $43.7 \%$ ), participant's suggestion to have UAI (UAI episode: $15.3 \%$; PAI episode: $6.5 \%$ ), anal sex took place during a weekday (UAI episode: $67.9 \%$; PAI episode: $54.0 \%$ ), condoms already placed at the venue where that anal intercourse took place (UAI episode: 70.7\%; PAI episode: $81.9 \%$ ), display of HIV prevention materials (e.g., posters) at the venue where anal sex took place (UAI episode: $41.4 \%$; PAI episode: $49.3 \%$ ), condoms possessed by the participant (UAI episode: $29.3 \%$; PAI episode: $37.2 \%$ ), and condoms possessed by the NRP (UAI episode: $11.6 \%$; PAI episode: $23.3 \%$ ).

3.2. Factors Distinguishing Episodes of UAI from Episodes of PAI. Higher likelihood of episodes involving UAI with NRP was associated with (1) one attribute of the NRP (perceived assertive or neutral personality $(\mathrm{OR}=2.18,95 \% \mathrm{CI}=1.07$ to 4.45 and $\mathrm{OR}=4.30,95 \% \mathrm{CI}=1.81$ to 10.17 ; reference group perceived passive personality)), (2) five situational variables (feeling tired before having anal sex $(\mathrm{OR}=2.73,95 \% \mathrm{CI}=$ 1.07 to 7.00$)$, feeling nervous before having anal sex (OR = $3.32,95 \% \mathrm{CI}=1.57$ to 7.06 ), perception that the partner was unlikely to or would never use condoms during anal sex with other men $(\mathrm{OR}=2.29,95 \% \mathrm{CI}=1.22$ to 4.28$)$, having the partner suggest UAI $(\mathrm{OR}=4.00,95 \% \mathrm{CI}=1.84$ to 8.68$)$, and having the participant suggest UAI $(\mathrm{OR}=2.90,95 \% \mathrm{CI}=$ 1.41 to 5.95$)$ ), and (3) one environmental/setting variable (the 
TABLE 2: Frequency distribution of the nonregular sex partners' (NRP) attributes.

\begin{tabular}{|c|c|c|}
\hline & $\begin{array}{c}\text { Episode involving condom use } \\
\text { (i.e., PAI) } \\
(n=215) \\
\text { Col } \% \\
\end{array}$ & $\begin{array}{l}\text { Episode involving no condom use } \\
\text { (i.e., UAI) } \\
\left(\begin{array}{c}n=215) \\
\text { Col } \%\end{array}\right.\end{array}$ \\
\hline \multicolumn{3}{|l|}{ Sociodemographic characteristics } \\
\hline \multicolumn{3}{|l|}{ Age (years) } \\
\hline$\leq 25$ & 23.7 & 31.2 \\
\hline $25-35$ & 45.1 & 41.9 \\
\hline $36-45$ & 9.8 & 11.2 \\
\hline$>46$ & 4.7 & 5.1 \\
\hline Do not know & 16.7 & 10.6 \\
\hline \multicolumn{3}{|l|}{ Where is he from? } \\
\hline Hong Kong & 82.8 & 82.8 \\
\hline Mainland & 4.7 & 7.4 \\
\hline Other countries & 11.2 & 8.4 \\
\hline Do not know & 1.4 & 1.4 \\
\hline \multicolumn{3}{|l|}{ Sexual experiences } \\
\hline \multicolumn{3}{|c|}{ How long did you know him before the intercourse? } \\
\hline On the same day & 55.8 & 59.5 \\
\hline$<0.5$ months & 19.5 & 20.5 \\
\hline $0.5-2$ months & 10.2 & 8.8 \\
\hline $2-6$ months & 5.6 & 4.7 \\
\hline$>6$ months & 8.8 & 6.5 \\
\hline \multicolumn{3}{|c|}{ Times of anal sex with the partner before } \\
\hline 0 times & 66.0 & 71.2 \\
\hline 1-2 times & 20.5 & 21.4 \\
\hline$\geq 3$ times & 13.5 & 7.4 \\
\hline \multicolumn{3}{|c|}{ Did you and your sex partner have equal sex experience? } \\
\hline About the same/do not know & 47.4 & 58.2 \\
\hline More experience & 35.8 & 26.0 \\
\hline Less experience & 16.7 & 15.8 \\
\hline \multicolumn{3}{|l|}{ Style of the partner } \\
\hline \multicolumn{3}{|l|}{ Physical appearance of the sex partner } \\
\hline Neutral & 38.1 & 44.7 \\
\hline Masculine & 47.9 & 47.0 \\
\hline Feminine & 14.0 & 8.3 \\
\hline \multicolumn{3}{|l|}{ Personality of the sex partner } \\
\hline Passive & 22.8 & 14.9 \\
\hline Assertive & 50.2 & 48.8 \\
\hline Neutral & 26.0 & 34.9 \\
\hline Do not know & 0.9 & 1.4 \\
\hline \multicolumn{3}{|l|}{ Liking and relationship } \\
\hline \multicolumn{3}{|l|}{ Attractiveness of the sex partner } \\
\hline Very attractive/attractive & 87.0 & 89.8 \\
\hline Not attractive/not very attractive & 12.5 & 9.7 \\
\hline Do not know & 0.5 & 0.5 \\
\hline
\end{tabular}


TABLE 2: Continued.

\begin{tabular}{|c|c|c|}
\hline & $\begin{array}{l}\text { Episode involving condom use } \\
\text { (i.e., PAI) } \\
(n=215) \\
\text { Col } \%\end{array}$ & $\begin{array}{l}\text { Episode involving no condom use } \\
\qquad \begin{array}{c}\text { (i.e., UAI) } \\
(n=215) \\
\text { Col } \%\end{array}\end{array}$ \\
\hline \multicolumn{3}{|c|}{ How much did you like the sex partner $(0-10) ?$} \\
\hline $1-3$ & 3.3 & 5.6 \\
\hline $4-6$ & 28.8 & 20.9 \\
\hline$\geq 7$ & 64.7 & 72.6 \\
\hline Do not know & 3.3 & 0.9 \\
\hline \multicolumn{3}{|c|}{ How much did the sex partner like you $(0-10)$ ? } \\
\hline $1-3$ & 1.9 & 4.2 \\
\hline $4-6$ & 17.2 & 24.6 \\
\hline$\geq 7$ & 65.6 & 52.6 \\
\hline Do not know & 15.3 & 18.6 \\
\hline \multicolumn{3}{|c|}{$\begin{array}{l}\text { Degree participant liked the sex partner more (difference in } 2 \\
\text { likeliness scores) }^{\#}\end{array}$} \\
\hline$<-3$ & 1.5 & 1.9 \\
\hline-1 to -3 & 20.9 & 17.6 \\
\hline 0 & 40.0 & 38.1 \\
\hline 1 to 3 & 20.9 & 20.5 \\
\hline$>3$ & 1.4 & 3.3 \\
\hline Do not know & 15.3 & 18.6 \\
\hline
\end{tabular}

\#It is the difference between the two likeliness scores: the degree of participant like the sex partner minus the degree of the sex partner like the participant.

anal sex episode took place on a weekday $(\mathrm{OR}=2.50,95 \%$ $\mathrm{CI}=1.49$ to 4.20$)$ ) (see Table 5).

Significantly lower likelihoods of episodes involving UAI with NRP were associated with (1) five NRP attributes (episodes involving NRP of $\leq 35$ years old or of unknown age $(\mathrm{OR}=0.52,95 \% \mathrm{CI}=0.28$ to 0.97 and $\mathrm{OR}=0.29$, $95 \%$ CI $=0.12$ to 0.70 ; reference group: $\leq 25$ years old), having had anal sex at least three times previously with the $\mathrm{NRP}(\mathrm{OR}=0.28,95 \% \mathrm{CI}=0.10$ to 0.75$)$, perception that he and the NRP possessed asymmetrical duration of sexual experience $(\mathrm{OR}=0.47,95 \% \mathrm{CI}=0.27$ to 0.79$)$, perception that the NRP looked feminine $(\mathrm{OR}=0.16,95 \% \mathrm{CI}=0.04$ to 0.57 ), and higher degree of liking toward the NRP (score $\geq 7$ out of 10$)(\mathrm{OR}=0.19,95 \% \mathrm{CI}=0.04$ to 0.81$))$, (2) six situational variables (perception that the participant had had UAI with another men in the last week (OR $=0.39,95 \% \mathrm{CI} \%$ $=0.18$ to 0.85$)$, discussion about condom use prior to anal sex $(\mathrm{OR}=0.29,95 \% \mathrm{CI}=0.16$ to 0.53$)$, perceived partner's liking in condom use $(\mathrm{OR}=0.11,95 \% \mathrm{CI}=0.05$ to 0.21$)$, the partner's suggestion to have PAI $(\mathrm{OR}=0.30,95 \% \mathrm{CI}=0.17$ to 0.52$)$, the participant's suggestion to have PAI $(\mathrm{OR}=0.19$, $95 \% \mathrm{CI}=0.10$ to 0.35$)$, and the participant planned to use condoms $(\mathrm{OR}=0.16,95 \% \mathrm{CI}=0.08$ to 0.30$)$ ), and (3) four environmental/setting variables (condoms already placed at the venue $(\mathrm{OR}=0.40,95 \% \mathrm{CI}=0.22$ to 0.71$)$, display of condom use promotion materials $(\mathrm{OR}=0.40,95 \% \mathrm{CI}=0.21$ to 0.76$)$, the participant possessed a condom $(\mathrm{OR}=0.45$, $95 \% \mathrm{CI}=0.24$ to 0.85$)$, and the NRP possessed a condom $(\mathrm{OR}=0.27,95 \% \mathrm{CI}=0.13$ to 0.56$)$ (Table 5$)$.
None of the variables belonging to the blocks of variables related to particular sexual behaviors, substance use, and the physical environment were found to be statistically significant in the conditional logistic regression analysis.

\section{Discussion}

This study presents in detail some situational factors that were associated with episodes of UAI with NRP. Some characteristics of the sexual episodes are interesting. A minority of the NRP was seen as HIV positive or at high risk of HIV infection but the majority of the NRP was seen to have other sex partners and not using condoms with such partners. Also, about $1 / 5$ and 1/7 of the participants had had UAI with another male partner during the week prior to the last episode of PAI and UAI with the NRP, respectively. Discussion about condom use with the NRP did not happen frequently. Condom availability appeared to be a barrier to engaging in PAI, as participants reported that neither they nor their NRP typically carried condoms with them. Overall, the participants reported being at high risk of HIV infection but not in a good position to prevent HIV infection.

Previous sexual experiences mattered in the prediction of UAI with NRP. The risk of UAI was lower for episodes involving NRP if the participants had had anal sex at least three times with that NRP. This suggested that it might be easier for the participants to negotiate condom use with a NRP that he had had sex with for several times in the 
TABLE 3: Frequency distributions of the situational variables.

\begin{tabular}{|c|c|c|}
\hline & $\begin{array}{c}\text { Episode involving condom use } \\
\text { (i.e., PAI) } \\
(n=215) \\
\text { Col } \% \\
\end{array}$ & $\begin{array}{l}\text { Episode involving no condom use } \\
\text { (i.e., UAI) } \\
\left(\begin{array}{c}n=215) \\
\text { Col } \%\end{array}\right.\end{array}$ \\
\hline \multicolumn{3}{|l|}{ Spirit prior to the episode of anal sex } \\
\hline \multicolumn{3}{|l|}{ Mood prior to the episode } \\
\hline Very good/good & 50.2 & 47.4 \\
\hline Normal & 44.2 & 45.1 \\
\hline Bad/very bad & 2.3 & 3.3 \\
\hline Do not know/cannot remember & 3.3 & 4.2 \\
\hline \multicolumn{3}{|l|}{ Tiredness prior to the episode } \\
\hline Very energetic/energetic & 46.0 & 42.4 \\
\hline Normal & 45.2 & 42.8 \\
\hline A bit tired/very tired & 6.0 & 10.7 \\
\hline Do not know/cannot remember & 2.8 & 4.2 \\
\hline \multicolumn{3}{|c|}{ Were you (the participant) nervous prior to the sex episode? } \\
\hline Not at all & 47.0 & 39.5 \\
\hline Not really & 39.1 & 36.3 \\
\hline A bit/very much & 11.2 & 20.9 \\
\hline Do not know & 2.8 & 3.3 \\
\hline \multicolumn{3}{|l|}{ Risk assessments } \\
\hline \multicolumn{3}{|c|}{$\begin{array}{l}\text { What do you think about the current risk of HIV infection of your } \\
\text { sex partner? }\end{array}$} \\
\hline Not infected & 43.7 & 45.6 \\
\hline Already infected/very high risk/high risk & 5.1 & 4.7 \\
\hline Very low risk/low risk & 51.2 & 49.7 \\
\hline \multicolumn{3}{|c|}{ What do you think about the current risk of STI of your sex partner? } \\
\hline Not infected & 43.3 & 44.7 \\
\hline Already infected/very high risk/high risk & 3.7 & 5.6 \\
\hline Very low risk/low risk & 53.0 & 49.7 \\
\hline \multicolumn{3}{|l|}{ Did his penis and anus look clean? } \\
\hline Yes & 73.5 & 70.7 \\
\hline \multicolumn{3}{|c|}{$\begin{array}{l}\text { How many male sex partners do you think your sex partner had in } \\
\text { the last month? }\end{array}$} \\
\hline None & 20.9 & 17.2 \\
\hline $1-5$ & 29.8 & 28.8 \\
\hline $6-10$ & 3.7 & 6.0 \\
\hline$>10$ & 1.9 & 1.9 \\
\hline Do not know & 43.7 & 46.0 \\
\hline \multicolumn{3}{|c|}{$\begin{array}{l}\text { How likely did you think he would use condoms with other male sex } \\
\text { partners? }\end{array}$} \\
\hline Very likely/moderate/no other partner & 58.6 & 50.2 \\
\hline Not likely/never & 41.4 & 49.8 \\
\hline \multicolumn{3}{|c|}{$\begin{array}{l}\text { One week before intercourse, did you have UAI with any other male } \\
\text { partner? }\end{array}$} \\
\hline Yes & 20.9 & 14.4 \\
\hline \multicolumn{3}{|c|}{ Did you and your sex partner have a third common sex partner? } \\
\hline Yes & 7.0 & 8.4 \\
\hline
\end{tabular}


TABLE 3: Continued.

Episode involving condom use Episode involving no condom use

$\begin{array}{ll}\text { (i.e., PAI) } & \text { (i.e., UAI) } \\ (n=215) & (n=215)\end{array}$

$\mathrm{Col} \%$

$\mathrm{Col} \%$

Communication \& planning about condom use

Did you discuss condom use with the partner before UAI/PAI?

Yes

Did sex partner suggest using a condom?

Yes

Did sex partner suggest NOT using a condom?

Yes

Did you suggest using a condom?

Yes

Did you suggest NOT using a condom?

Yes

Did you plan to use a condom during that sexual encounter? Yes

Did you perceive that the sex partner would like to use a condom? Yes

Particular sexual behavior

Degree of sexual compulsivity of participant (0-10)

$1-5$

$6-10$

Degree of sexual compulsivity of sex partner (0-10)

$1-5$

6-10

Participant's sexual position

Insertive

Receptive

Both

Do not know

One month before the episode, did you have any problem with sexual dysfunction?

Yes

During the intercourse, how many sex partners were there?

More than 1

Any erotic activities involved?

Yes (at least one)*

Whether money transaction was involved in the episode?

No

Yes

Cannot remember

Substance use

Whether you (the participant) or sex partner drank alcohol before the anal sex episode? (if either man drank answer is classified as "yes") 
TABLE 3: Continued.

Episode involving condom use Episode involving no condom use

$\begin{array}{ll}\text { (i.e., PAI) } & \text { (i.e., UAI) } \\ (n=215) & (n=215)\end{array}$

$\mathrm{Col} \%$

$\mathrm{Col} \%$

Whether you or sex partner used Viagra or psychoactive substances

before the anal sex episode?

Yes

2.3

4.2

*Erotic activities include watching sex movie before UAI, reading pornographic magazines, the use of sex toys, SM behaviour, and wearing special outfits.

TABLE 4: Frequency distributions of environmental/settings variables.

\begin{tabular}{|c|c|c|}
\hline & $\begin{array}{l}\text { Episode involving condom use } \\
\text { (i.e., PAI) } \\
(n=215) \\
\text { Col } \% \\
\end{array}$ & $\begin{array}{l}\text { Episode involving no condom use } \\
\text { (i.e., UAI) } \\
\left(\begin{array}{c}n=215) \\
\text { Col } \%\end{array}\right.\end{array}$ \\
\hline \multicolumn{3}{|l|}{ Timing and location } \\
\hline \multicolumn{3}{|l|}{ Location of the episode } \\
\hline Hong Kong & 89.3 & 90.2 \\
\hline Overseas & 10.7 & 9.8 \\
\hline \multicolumn{3}{|l|}{ Happened during a weekend? } \\
\hline Weekend & 46.0 & 32.1 \\
\hline Weekday & 54.0 & 67.9 \\
\hline \multicolumn{3}{|l|}{ Hour of the day when it occurred? } \\
\hline Morning/afternoon & 32.1 & 30.2 \\
\hline Evening & 67.9 & 69.8 \\
\hline \multicolumn{3}{|l|}{ Did the episode occur at home setting? } \\
\hline Yes (participant or sex partner's home) & 34.4 & 29.7 \\
\hline No (e.g., hotel, sauna, etc.) & 65.5 & 70.2 \\
\hline \multicolumn{3}{|l|}{ Availability of condoms } \\
\hline \multicolumn{3}{|l|}{ Were condoms already available at the venue? } \\
\hline Yes & 81.9 & 70.7 \\
\hline \multicolumn{3}{|l|}{ Were there free condoms available at the venue? } \\
\hline Yes & 60.9 & 56.7 \\
\hline \multicolumn{3}{|c|}{$\begin{array}{l}\text { Were there any notices (e.g., posters) promoting condom use at } \\
\text { the venue then? }\end{array}$} \\
\hline Yes & 49.3 & 41.4 \\
\hline \multicolumn{3}{|l|}{ Were you carrying a condom with him? } \\
\hline Yes & 37.2 & 29.3 \\
\hline \multicolumn{3}{|c|}{ Was the sex partner carrying a condom with him? } \\
\hline Yes & 23.3 & 11.6 \\
\hline \multicolumn{3}{|l|}{ Physical environment } \\
\hline \multicolumn{3}{|l|}{ Was it a dark environment? } \\
\hline Yes & 70.2 & 68.4 \\
\hline \multicolumn{3}{|l|}{ Was there a place for shower? } \\
\hline Yes & 97.7 & 96.7 \\
\hline \multicolumn{3}{|l|}{ Was the hygiene relatively poor? } \\
\hline Yes & 7.0 & 7.9 \\
\hline
\end{tabular}


TABLE 5: Factors associated with UAI involving male NRP.

\begin{tabular}{|c|c|c|c|}
\hline & $\begin{array}{l}\text { Episode involving condom use } \\
\qquad(n=215)\end{array}$ & $\begin{array}{l}\text { Episode involving no condom use } \\
\qquad(n=215)\end{array}$ & Matched OR \\
\hline & Row \% & Row \% & \\
\hline \multicolumn{4}{|l|}{ Sociodemographic characteristics } \\
\hline \multicolumn{4}{|l|}{ Age (years) } \\
\hline$\leq 25$ & 43.2 & 56.8 & 1 \\
\hline $26-35$ & 51.9 & 48.1 & $0.52(0.28-0.97)^{*}$ \\
\hline $36-45$ & 46.7 & 53.3 & $0.66(0.26-1.72)$ \\
\hline$>46$ & 47.6 & 52.4 & $0.93(0.13-6.50)$ \\
\hline Do not know & 61.0 & 39.0 & $0.29(0.12-0.70)^{* *}$ \\
\hline \multicolumn{4}{|l|}{ Sexual experiences } \\
\hline \multicolumn{4}{|c|}{ Times of anal sex with the sex partner before } \\
\hline 0 times & 48.1 & 51.9 & 1 \\
\hline 1-2 times & 48.9 & 51.1 & $1.02(0.49-2.12)$ \\
\hline$\geq 3$ times & 64.4 & 35.6 & $0.28(0.10-0.75)^{*}$ \\
\hline \multicolumn{4}{|c|}{$\begin{array}{l}\text { Did your sex partner have equal sex experience } \\
\text { as you? }\end{array}$} \\
\hline About the same/do not know & 44.9 & 55.1 & 1 \\
\hline More experience/less experience & 55.7 & 44.3 & $0.47(0.27-0.79)^{* *}$ \\
\hline \multicolumn{4}{|l|}{ Style of the partner } \\
\hline \multicolumn{4}{|l|}{ Physical appearance of sex partner } \\
\hline Neutral & 46.1 & 53.9 & 1 \\
\hline Masculine & 50.5 & 49.5 & $0.67(0.38-1.20)$ \\
\hline Feminine & 62.5 & 37.5 & $0.16(0.04-0.57)^{* *}$ \\
\hline \multicolumn{4}{|l|}{ Personality of sex partner } \\
\hline Passive & 60.5 & 39.5 & 1 \\
\hline Assertive & 50.7 & 49.3 & $2.18(1.07-4.45)^{*}$ \\
\hline Neutral & 42.7 & 57.3 & $4.30(1.81-10.17)^{* * *}$ \\
\hline Do not know & 40.0 & 60.0 & $4.48(0.35-57.46)$ \\
\hline \multicolumn{4}{|l|}{ Liking \& relationship } \\
\hline \multicolumn{4}{|l|}{ How much did the sex partner like you? } \\
\hline $1-3$ & 30.8 & 69.2 & 1 \\
\hline $4-6$ & 41.1 & 58.9 & $0.60(0.14-2.69)$ \\
\hline$\geq 7$ & 55.5 & 44.5 & $0.19(0.04-0.81)^{*}$ \\
\hline Do not know & 45.2 & 54.8 & $0.76(0.17-3.47)$ \\
\hline \multicolumn{4}{|l|}{ Spirit prior to the episode of anal sex } \\
\hline \multicolumn{4}{|l|}{ Tiredness before the episode } \\
\hline Very energetic/energetic/normal & 51.7 & 48.3 & 1 \\
\hline A bit tired/very tired & 36.1 & 63.9 & $2.73(1.07-7.00)^{*}$ \\
\hline Do not know/cannot remember & 40.0 & 60.0 & $1.61(0.57-4.56)$ \\
\hline \multicolumn{4}{|c|}{$\begin{array}{l}\text { Were you (the participant) nervous prior to the } \\
\text { sex episode? }\end{array}$} \\
\hline Not at all & 54.3 & 45.7 & 1 \\
\hline Not really & 51.9 & 48.1 & $1.33(0.75-2.33)$ \\
\hline A bit/very & 34.8 & 65.2 & $3.32(1.57-7.06)^{* *}$ \\
\hline Do not know & 46.2 & 53.8 & $1.61(0.47-5.57)$ \\
\hline
\end{tabular}


TABle 5: Continued.

\begin{tabular}{|c|c|c|c|}
\hline & $\begin{array}{l}\text { Episode involving condom use } \\
\qquad \begin{array}{c}(n=215) \\
\text { Row } \%\end{array}\end{array}$ & $\begin{array}{c}\text { Episode involving no condom use } \\
\qquad \begin{array}{c}(n=215) \\
\text { Row } \%\end{array}\end{array}$ & Matched OR \\
\hline \multicolumn{4}{|l|}{ Risk assessments } \\
\hline \multicolumn{4}{|l|}{$\begin{array}{l}\text { One week before intercourse, did you have UAI } \\
\text { with any other male partner? }\end{array}$} \\
\hline No & 48.0 & 52.0 & 1 \\
\hline Yes & 59.2 & 40.8 & $0.39(0.18-0.85)^{*}$ \\
\hline \multicolumn{4}{|l|}{$\begin{array}{l}\text { How likely did you think he would be to use } \\
\text { condoms with other male sex partners? }\end{array}$} \\
\hline Very likely/moderate/no other partners & 53.8 & 46.2 & 1 \\
\hline Not likely/never & 45.4 & 54.6 & $2.29(1.22-4.28)^{*}$ \\
\hline \multicolumn{4}{|l|}{ Communication \& planning about condom use } \\
\hline \multicolumn{4}{|c|}{$\begin{array}{l}\text { Did you discuss condom use with partner before } \\
\text { UAI? }\end{array}$} \\
\hline No & 44.4 & 55.6 & 1 \\
\hline Yes & 63.5 & 36.5 & $0.29(0.16-0.53)^{* * *}$ \\
\hline \multicolumn{4}{|c|}{$\begin{array}{l}\text { Did you perceive that the sex partner would like } \\
\text { to use a condom? }\end{array}$} \\
\hline No & 36.9 & 63.1 & 1 \\
\hline Yes & 76.2 & 23.8 & $0.11(0.05-0.21)^{* * *}$ \\
\hline \multicolumn{4}{|l|}{ Did your sex partner suggest using a condom? } \\
\hline No & 44.1 & 55.9 & 1 \\
\hline Yes & 67.6 & 32.4 & $0.30(0.17-0.52)^{* * *}$ \\
\hline \multicolumn{4}{|l|}{$\begin{array}{l}\text { Did your sex partner suggest NOT using a } \\
\text { condom? }\end{array}$} \\
\hline No & 49.0 & 51.0 & 1 \\
\hline Yes & 35.0 & 65.0 & $4.00(1.84-8.68)^{* * *}$ \\
\hline \multicolumn{4}{|l|}{ Did you plan to use a condom? } \\
\hline No & 28.7 & 71.3 & 1 \\
\hline Yes & 59.9 & 40.1 & $0.16(0.08-0.30)^{* * *}$ \\
\hline \multicolumn{4}{|l|}{ Did you suggest using a condom? } \\
\hline No & 41.3 & 58.7 & 1 \\
\hline Yes & 68.6 & 31.4 & $0.19(0.10-0.35)^{* * *}$ \\
\hline \multicolumn{4}{|l|}{ Did you suggest NOT using a condom? } \\
\hline No & 52.5 & 47.5 & 1 \\
\hline Yes & 29.8 & 70.2 & $2.90(1.41-5.95)^{* *}$ \\
\hline \multicolumn{4}{|l|}{ Timing \& location } \\
\hline \multicolumn{4}{|l|}{ Happen during weekend? } \\
\hline Weekend & 58.9 & 41.1 & 1 \\
\hline Weekday & 44.3 & 55.7 & $2.50(1.49-4.20)^{* * *}$ \\
\hline \multicolumn{4}{|l|}{ Availability of condoms } \\
\hline \multicolumn{4}{|l|}{ Were condoms already available at the venue? } \\
\hline No & 38.2 & 61.8 & 1 \\
\hline Yes & 53.7 & 46.3 & $0.40(0.22-0.71)^{* *}$ \\
\hline \multicolumn{4}{|c|}{$\begin{array}{l}\text { Were there any notices (e.g., posters) promoting } \\
\text { condom use at the venue then? }\end{array}$} \\
\hline No & 46.4 & 53.6 & 1 \\
\hline Yes & 54.4 & 45.6 & $0.40(0.21-0.76)^{*}$ \\
\hline
\end{tabular}


TABle 5: Continued.

\begin{tabular}{lccc}
\hline & $\begin{array}{c}\text { Episode involving condom use } \\
(n=215) \\
\text { Row } \%\end{array}$ & $\begin{array}{c}\text { Episode involving no condom use } \\
(n=215) \\
\text { Row \% }\end{array}$ & $\begin{array}{c}\text { Matched OR } \\
\text { Were you carrying a condom? }\end{array}$ \\
No & 47.0 & 53.0 & 1 \\
Yes & 55.9 & 44.1 & $0.45(0.24-0.85)^{*}$ \\
Was the sex partner carrying a condom with & & & 53.5 \\
him? & 46.5 & 33.3 & 1 \\
No & 66.7 & $0.27(0.13-0.56)^{* * *}$ \\
Yes
\end{tabular}

${ }^{*} P<0.05 ;{ }^{* *} P<0.01 ;{ }^{* * *} P<0.001$

\# Adjusted for location of sex episode.

past. Episodes involving asymmetrical duration of sexual experiences between the participant and the NRP were also associated with lower risk of UAI. It is possible the participant or the NRP would be more cautious when facing a sex partner who is more or less sexually experienced. The exact reasons behind the UAI are not clear. As a limitation, we did not separate higher versus lower level of sexual experience as compared to that of the NRP.

Those NRP perceived as being feminine, being perceived as having a less assertive personality, or being liked by the participant were less likely to engage in UAI. Thus, HIV prevention education might give special attention to MSM who look masculine or those who have a more assertive personality. There is evidence that, among consistent and inconsistent condom users, being sexually attracted to a partner is a risk factor for unprotected sex [25]; however, the findings of the current study are not consistent with this, suggesting that factors discriminating between episodes of UAI and PAI with NRP among MSM who are inconsistent condom users may be different from those discriminating between consistent and inconsistent condom users among all MSM.

It is important to note that almost all the situational factors related to condom negotiation and the perception that the partner liked to use condoms were significant protective factors of episodes of UAI with NRP, corroborating with results obtained from studies discriminating between consistent and inconsistent condom users among all MSM [26]. Therefore, HIV prevention attempting to reduce UAI among MSM who are inconsistent condom users with NRP should focus on situational factors. As the perception that the NRP disliked using condoms with other male sex partners was also a significant factor, MSM with NRP should be reminded not to assume that the NRP would not want to use condoms, as such perceptions may be inaccurate while preference on condom use is relative and changeable. Instead, they should be encouraged to discuss condom use explicitly and consistently with the NRP.

Of equal importance is the protective effect of condom availability, which corroborates literature discriminating consistent versus inconsistent condom users among MSM [6, 27]. The results of the current study have practical implications, in that it seems useful to place and to distribute condoms at the venue where anal sex commonly takes place. The participants' possession of condoms is another protective factor against episodes of UAI with NRP. HIV prevention programs should therefore attempt to create a norm that it is socially acceptable and likely beneficial for sexually active MSM to always carry a condom with them. Small, stylish, and high quality capsules (e.g., key chains) to carry condoms can be designed and distributed to MSM. Many intervention programs, such as those involving condom distribution exercises, have tried to increase condom availability [28] but very few programs have reminded MSM to always carry condoms with them. This attempt would be a complement to other efforts promoting condom availability among MSM having anal intercourses with NRP.

In addition, based on the current results, seeing reminders promoting condom use displayed at venues where sexual encounters occur might reduce the risk of UAI with NRP. Venues such as gay saunas and hotels should therefore ensure that there is a supply of condoms and display such posters or reminders that remind MSM to use condoms at that venue. These posters may thus serve as cue to action, which is a construct of the Health Belief Model that has shown to be significantly associated with many health-related behaviors, including condom use [29]. In HIV prevention programs, MSM who are inconsistent condom users may also be provided with reminders about every-time condom use, items that they can wear (e.g., pins and bracelets) or be placed at home (e.g., photo frame, towels, and bathroom utilities) that can serve as cues to action to avoid UAI. They can also choose their own items, which may not need to be specifically related to HIV prevention to avoid stigmatization.

One surprising result from the current study is that sexual episodes taking place during a weekday and tiredness and nervous feelings prior to anal sex with NRP were risk factors of UAI with NRP. MSM who are inconsistent condom users with NRP should be reminded about the increased risks of UAI when they are exposed to these risky situations. In practice, more venue-based (e.g., gay saunas) HIV prevention outreach activities could be conducted during weekdays. Special midweek reminders may also be flagged at such 
venues. MSM should also be reminded of the importance of foreplay to reduce any nervous feelings prior to anal intercourse. At gay saunas, for instance, MSM may be advised to have a massage before engaging in sexual intercourse so as to reduce tiredness and anxiety. These are ideas that have not yet been investigated, as many of these factors leading to UAI have not been investigated in previous studies of consistent and inconsistent condom users.

Previous studies have shown that the use of alcohol and substances was associated with UAI but mixed results were obtained [30, 31]. It is interesting that our data did not find alcohol use to be a risk factor of episodes of UAI with NRP. The prevalence of substance use was rather low and no significant associations with UAI were found. Other potentially important factors were also found to be nonsignificantly related to UAI, including perception that the NRP have contracted HIV/STI and perceived number of sex partners of the NRP. Such findings are informative, as they remind healthcare workers that MSM may have UAI with NRP even if they are aware that such intercourses may involve high risk of HIV/STI transmission. It implies that HIV prevention attempting to raise relevant risk perceptions, a common approach, would be unlikely to be effective in prevention programs targeting MSM who are inconsistent condom users. The associations between risk perception and condom use reported in studies discriminating consistent and inconsistent condom users were mixed [32].

Furthermore, while variables related to the physical environment (e.g., shower facilities and hygiene), sexual compulsivity, sexual positions were found to be significantly associated with UAI in traditional studies discriminating consistent and inconsistent condom users [33-37], such variables were nonsignificant in this study. This discrepancy confirms the need to consider a different set of factors for designing HIV prevention campaigns targeting MSM who are inconsistent condom users with NRP.

In sum, factors that have been shown to discriminate between consistent condom users and inconsistent condom users may not be applicable to understanding why MSM who are inconsistent condom users sometimes but not always use condoms during anal sex with NRP. It is important to ensure that those distinguishing factors not be overemphasized when designing HIV interventions. Instead, situational factors such as those found in this study should be considered. In addition, more research in this area is warranted.

The case-crossover study design has the benefit of minimizing between individual confounders as each individual serves as his own control [16]. The study, however, also has some limitations. First, the data used was based on selfreported ones and was hence subject to recall bias. Second, the time since the last episodes of UAI and PAI may have varied between individuals; the time interval between the UAI and the PAI was not uniform across individuals. Third, there may have been multiple incidences of UAI and PAI within the participants. The association with UAI may vary, depending on which pair of episodes were selected. We chose only the most recent pair for data analysis and assumed high consistency in characteristics associated with different episodes of UAI and different episodes of PAI, which may not always be true. Fourth, information about the frequency of UAI and PAI was not collected in this study. The design allows for investigation of event-specific factors among inconsistent condom users. However, the design also treated inconsistent condom users with NRP as a homogeneous group while inconsistent condom users with NRP may in fact vary in frequency of intercourse and frequency of UAI. Fifth, some limitations exist with regard to measurements. In this study, we asked whether the participants felt nervous in general prior to the last episode of UAI or PAI of concern. We did not ask the participant to specify the reasons for their nervous feeling and whether this feeling was related to condom use, but it would be difficult to discern the cause of such feelings. The variable of sexual dysfunction referred to perceived sexual performance in the last month; the situation may vary over time but it might not be strictly regarded as an eventspecific situational factor. Another limitation was related to variable selection. Our variable selection was based on a literature search of traditional cross-sectional studies as the few case-crossover studies on HIV-related behaviors used only a narrow range of independent variables $[38,39]$. In addition, some inconsistent condom users may not admit their condom use status or the fact that they had had sex with NRP due to social desirability. Selection bias may also exist due to the absence of a sampling frame, although previous studies on HIV-related behaviors among MSM have also used similar mixed-method recruitment strategies [18-20, 40, 41].

In sum, we found that availability and possession of condoms, condom negotiation, and planning to use condoms were event-specific protective factors against episodes of UAI with NRP among MSM who were inconsistent condom users. We also discovered some new situational risk factors such as anal sex taking place during the weekday and tiredness prior to anal sex and have identified other situational and environmental protective or risk factors such as display of reminders promoting condom use, home settings, travel, and nervous feelings. We have further found that factors such as the physical environments, particular sexual behaviors, and assessment of the partner's risk were nonsignificant. These findings provide important insights for designing new HIV prevention targeting inconsistent condom users by putting more emphasis on event-specific factors. Such factors may partially explain why condoms are sometimes but not always used by MSM.

\section{Conflict of Interests}

There is no conflict of interests for any author.

\section{Acknowledgments}

The research team would like to thank all participants that took part in the study. Thanks are extended to all others who had helped in the entire data collection period. The study was supported by the Research Fund for the Control of Infectious Diseases, Health, Welfare, and Food Bureau, Hong Kong Special Administrative Region (Project ref.: \#09080032). 


\section{References}

[1] UNAIDS, HIV and Men Who Have Sex with Men in Asia and the Pacific, UNAIDS, Geneva, Switzerland, 2006.

[2] Ministry of Health People's Republic of China, Joint United Nations Programme on HIV/AIDS, World Health Organization. 2009 Estimates for the HIV/AIDS Epidemic in China, 2010.

[3] H. Yang, C. Hao, X. Huan et al., "HIV incidence and associated factors in a cohort of men who have sex with men in Nanjing, China," Sexually Transmitted Diseases, vol. 37, no. 4, pp. 208-213, 2010.

[4] J. Xu, M. Zhang, K. Brown et al., "Syphilis and HIV seroconversion among a 12-month prospective cohort of men who have sex with men in Shenyang, China," Sexually Transmitted Diseases, vol. 37, no. 7, pp. 432-439, 2010.

[5] Special Preventive Programme, PRiSM-HIV Prevalence and Risk Behavioural Survey for Men Who Have Sex with Men in Hong Kong 2008, Centre for Health Protection, Department of Health, HKSAR, 2009.

[6] J. T. F. Lau, W. Cai, H. Y. Tsui et al., "Prevalence and correlates of unprotected anal intercourse among Hong Kong men who have sex with men traveling to Shenzhen, China," AIDS and Behavior, vol. 17, no. 4, pp. 1395-1405, 2013.

[7] S. H. Lim, A. R. Bazazi, C. Sim, M. Choo, F. L. Altice, and A. Kamarulzaman, "High rates of unprotected anal intercourse with regular and casual partners and associated risk factors in a sample of ethnic Malay men who have sex with men (MSM) in Penang, Malaysia," Sexually Transmitted Infections, vol. 89, pp. 642-649, 2013.

[8] G. Mansergh, S. Naorat, R. Jommaroeng et al., "Inconsistent condom use with steady and casual partners and associated factors among sexually-active men who have sex with men in Bangkok, Thailand," AIDS and Behavior, vol. 10, no. 6, pp. 743751, 2006.

[9] X. Li, W. Shi, D. Li et al., "Predictors of unprotected sex among men who have sex with men in Beijing, China," Southeast Asian Journal of Tropical Medicine and Public Health, vol. 39, no. 1, pp. 99-108, 2008.

[10] J. T. F. Lau, W. Cai, H. Y. Tsui et al., "Unprotected anal intercourse behavior and intention among male sex workers in Shenzhen serving cross-boundary male clients coming from Hong Kong, China-prevalence and associated factors," AIDS Care, vol. 24, no. 1, pp. 59-70, 2012.

[11] J. T. Lau, T. J. Feng, X. L. Liu et al., "Associations between cognitive, sociocontextual, and affective variables and unprotected anal intercourse among men who have sex with mena comparative study conducted in two Chinese cities," BioMed Research International, vol. 2014, Article ID 970975, 9 pages, 2014.

[12] S. Kippax, J. Crawford, P. Rodden, and J. Noble, "Predictors of unprotected male-to-male anal intercourse with casual partners in a national sample," Australian Journal of Public Health, vol. 19, no. 2, pp. 132-138, 1995.

[13] J. Gu, H. Xu, J. T. Lau et al., "Situation-specific factors predicting nonadherence to methadone maintenance treatment: a crosssectional study using the case-crossover design in Guangzhou, China," AIDS Care, pp. 1-6, 2014.

[14] J. F. Liang, Y. T. Chen, J. L. Fuh et al., "Proton pump inhibitor-related headaches: a nationwide population-based case-crossover study in Taiwan," Cephalalgia, 2014.
[15] G. R. Seage III, S. Holte, M. Gross et al., "Case-crossover study of partner and situational factors for unprotected sex," Journal of Acquired Immune Deficiency Syndromes, vol. 31, no. 4, pp. 432439, 2002.

[16] L. Warner, M. Macaluso, H. D. Austin et al., "Application of the case-crossover design to reduce unmeasured confounding in studies of condom effectiveness," The American Journal of Epidemiology, vol. 161, no. 8, pp. 765-773, 2005.

[17] M. Maclure, "The case-crossover design: a method for studying transient effects on the risk of acute events," The American Journal of Epidemiology, vol. 133, no. 2, pp. 144-153, 1991.

[18] J. Gu, J. T. F. Lau, and H. Tsui, "Psychological factors in association with uptake of voluntary counselling and testing for HIV among men who have sex with men in Hong Kong," Public Health, vol. 125, no. 5, pp. 275-282, 2011.

[19] J. T. Lau, "A comprehensive study for utilization of VCT services in MSM-prevalence, patterns, needs and associated factors," Report submitted to the Hong Kong Council for the AIDS Trust Fund (\#SPF028), 2009.

[20] H. Y. Tsui and J. T. F. Lau, "Comparison of risk behaviors and socio-cultural profile of men who have sex with men survey respondents recruited via venues and the internet," BMC Public Health, vol. 10, article 232, 2010.

[21] Y. Song, X. Li, L. Zhang et al., "HIV-testing behavior among young migrant men who have sex with men (MSM) in Beijing, China," AIDS Care-Psychological and Socio-Medical Aspects of AIDS/HIV, vol. 23, no. 2, pp. 179-186, 2011.

[22] Y. Xiao, X. Ding, C. Li, J. Liu, J. Sun, and Y. Jia, "Prevalence and correlates of HIV and syphilis infections among men who have sex with men in chongqing municipality, China," Sexually Transmitted Diseases, vol. 36, no. 10, pp. 647-656, 2009.

[23] J. T. F. Lau, M. Lau, A. Cheung, and H. Y. Tsui, "A randomized controlled study to evaluate the efficacy of an Internet-based intervention in reducing HIV risk behaviors among men who have sex with men in Hong Kong," AIDS Care: Psychological and Socio-Medical Aspects of AIDS/HIV, vol. 20, no. 7, pp. 820-828, 2008.

[24] J. T. Lau, H. Y. Tsui, and M. M. Lau, "A pilot clustered randomized control trial evaluating the efficacy of a networkbased HIV peer-education intervention targeting men who have sex with men in Hong Kong, China," AIDS Care, vol. 25, no. 7, pp. 812-819, 2013.

[25] G. Lambert, J. Cox, T. S. Hottes et al., "Correlates of unprotected anal sex at last sexual episode: analysis from a surveillance study of men who have sex with men in Montreal," AIDS and Behavior, vol. 15, no. 3, pp. 584-595, 2011.

[26] P. A. Wilson, R. M. Díaz, H. Yoshikawa, and P. E. Shrout, "Drug use, interpersonal attraction, and communication: situational factors as predictors of episodes of unprotected anal intercourse among Latino gay men," AIDS and Behavior, vol. 13, no. 4, pp. 691-699, 2009.

[27] N. Y. Ko, H. C. Lee, C. C. Hung et al., "Effects of structural intervention on increasing condom availability and reducing risky sexual behaviours in gay bathhouse attendees," AIDS Care, vol. 21, no. 12, pp. 1499-1507, 2009.

[28] Z. Huang, M. Wang, L. Fu et al., "Intervention to increase condom use and HIV testing among men who have sex with men in China: a meta-analysis," AIDS Research and Human Retroviruses, vol. 29, no. 3, pp. 441-448, 2013.

[29] I. M. Rosenstock, V. J. Stretcher, and M. H. Becker, "The health belief model and HIV risk behavior change," in Preventing 
AIDS: Theories and Methods of Behavioral Interventions, R. J. DiClemente and J. L. Peterson, Eds., pp. 5-25, Plenum Press, New York, NY, USA, 1994.

[30] B. A. Koblin, M. A. Chesney, M. J. Husnik et al., "High-risk behaviors among men who have sex with men in 6 US cities: baseline data from the explore study," The American Journal of Public Health, vol. 93, no. 6, pp. 926-932, 2003.

[31] J. L. Martin, "Drug use and unprotected anal intercourse among gay men," Health Psychology, vol. 9, no. 4, pp. 450-465, 1990.

[32] M. Gerrard, F. X. Gibbons, and B. J. Bushman, "Relation between perceived vulnerability to HIV and precautionary sexual behavior," Psychological Bulletin, vol. 119, no. 3, pp. 390409, 1996.

[33] J. Bancroft, L. Carnes, and E. Janssen, "Unprotected anal intercourse in HIV-Positive and HIV-negative gay men: the relevance of sexual arousability, mood, sensation seeking, and erectile problems," Archives of Sexual Behavior, vol. 34, no. 3, pp. 299-305, 2005.

[34] C. Grov, J. T. Parsons, and D. S. Bimbi, "Sexual compulsivity and sexual risk in gay and bisexual men," Archives of Sexual Behavior, vol. 39, no. 4, pp. 940-949, 2010.

[35] V. Hemmige, H. Snyder, C. Liao et al., "Sex position, marital status, and HIV risk among indian men who have sex with men: clues to optimizing prevention approaches," AIDS Patient Care and STDs, vol. 25, no. 12, pp. 725-734, 2011.

[36] J. T. F. Lau, J. Zhao, X. Wu, J. Gu, and C. Hao, "Gay saunas and the risks of HIV and syphilis transmissions in China-results of a meta-analysis," Journal of Sexual Medicine, vol. 10, no. 3, pp. 642-652, 2013.

[37] W. J. Reidy, F. Spielberg, R. Wood, D. Binson, W. J. Woods, and G. M. Goldbaum, "HIV risk associated with gay bathhouses and sex clubs: findings from 2 seattle surveys of factors related to HIV and sexually transmitted infections," The American Journal of Public Health, vol. 99, supplement 1, pp. S165-S172, 2009.

[38] B. A. Koblin, C. Murrill, M. Camacho et al., "Amphetamine use and sexual risk among men who have sex with men: results from the National HIV Behavioral Surveillance study-New York City," Substance Use and Misuse, vol. 42, no. 10, pp. 16131628, 2007.

[39] H. V. Tieu, C. Murrill, G. Xu, and B. A. Koblin, "Sexual partnering and HIV risk among black men who have sex with men: New York city," The Journal of Urban Health, vol. 87, no. 1, pp. 113-121, 2010.

[40] M. I. Fernández, T. Perrino, J. B. Collazo et al., "Surfing new territory: club-drug use and risky sex among Hispanic men who have sex with men recruited on the internet," Journal of Urban Health, vol. 82, no. 1, pp. i79-i88, 2005.

[41] Y. Hidaka, S. Ichikawa, J. Koyano et al., "Substance use and sexual behaviours of Japanese men who have sex with men: a nationwide internet survey conducted in Japan," BMC Public Health, vol. 6, article 239, 2006. 


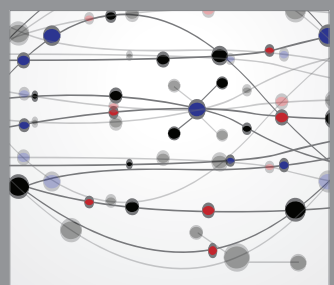

The Scientific World Journal
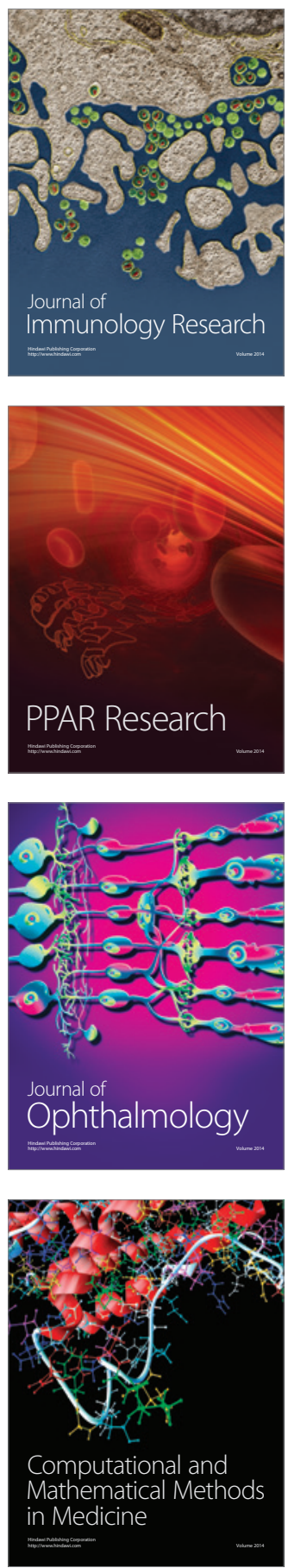

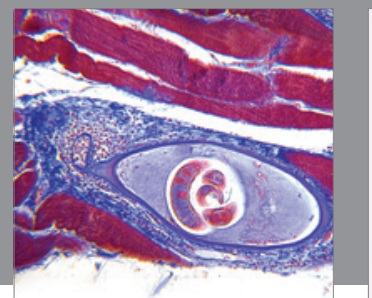

Gastroenterology

Research and Practice
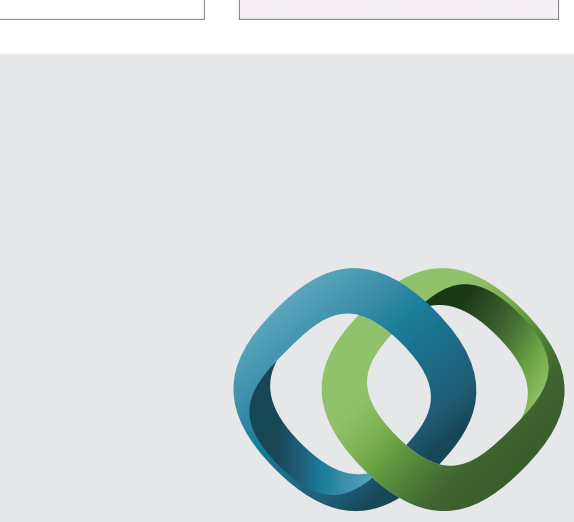

\section{Hindawi}

Submit your manuscripts at

http://www.hindawi.com
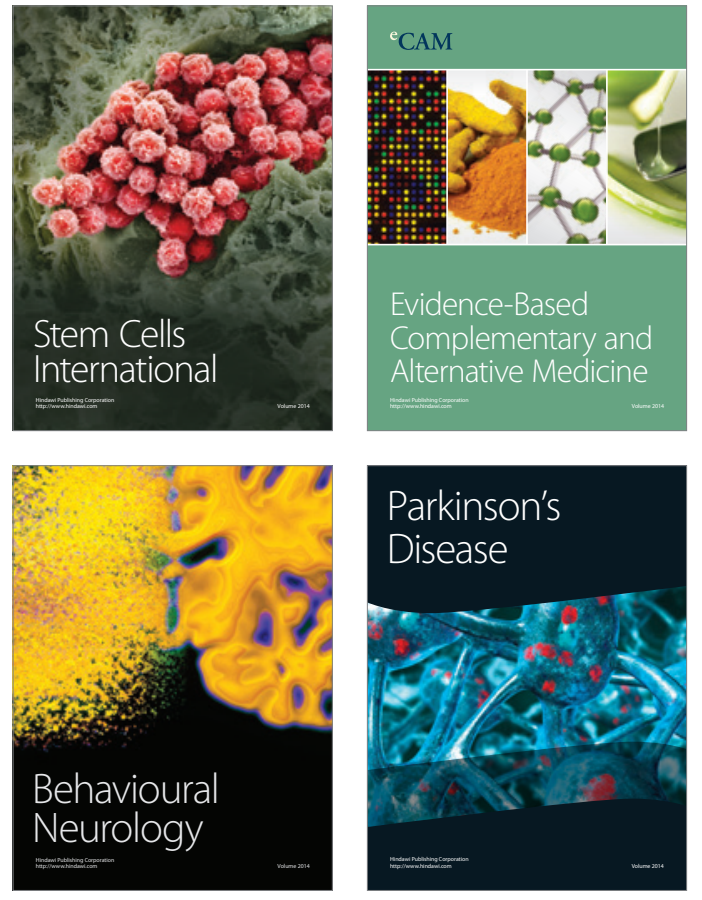
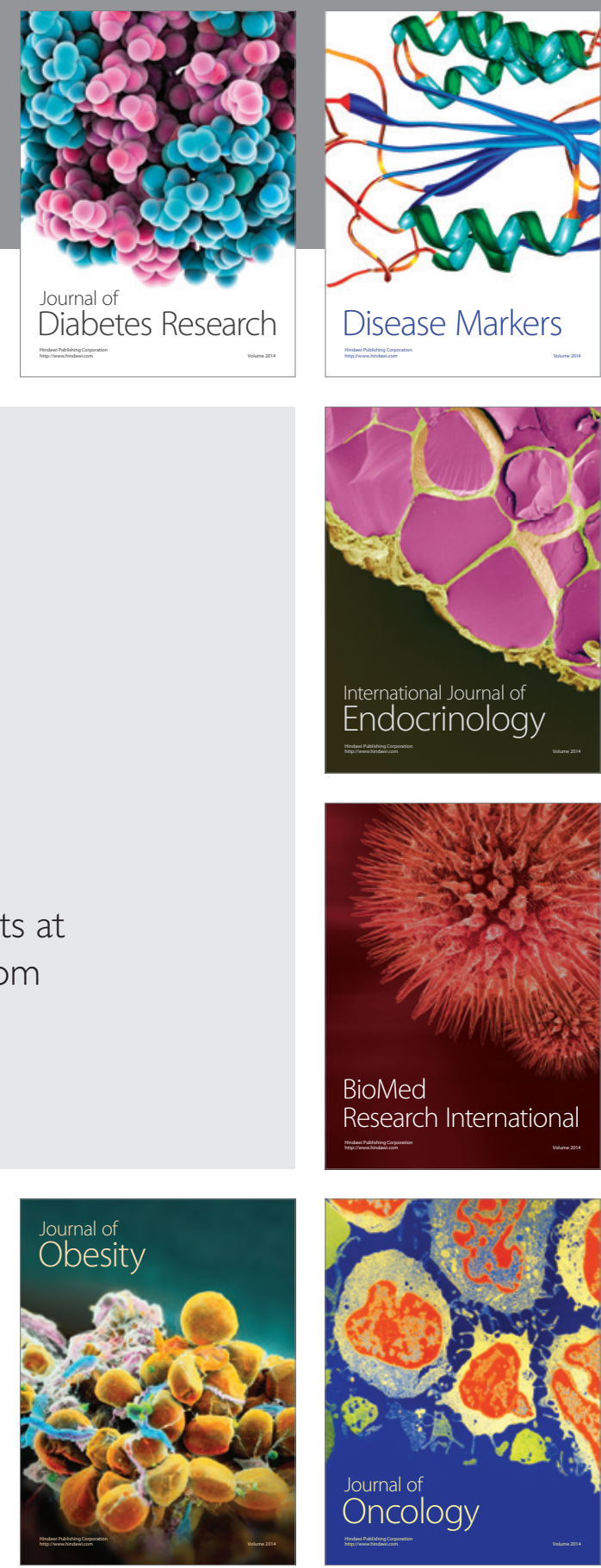

Disease Markers
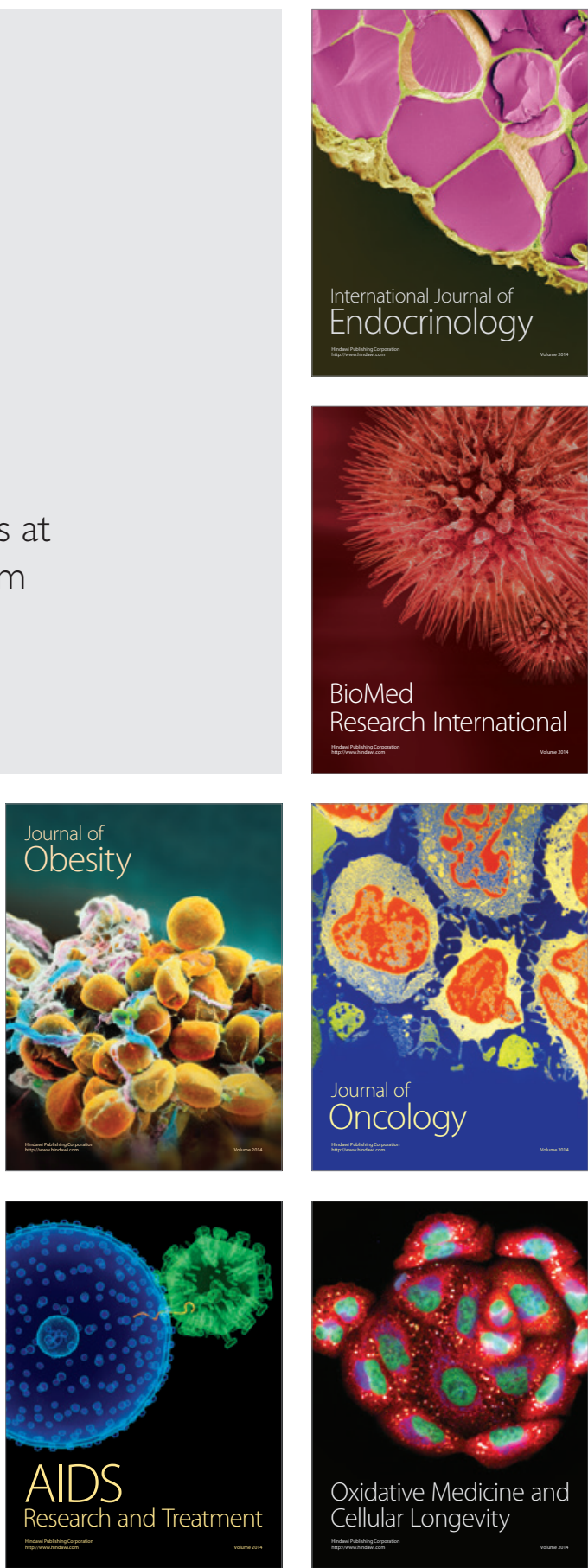